\title{
Institutional NIH Research Funding and a Culture of Support for Family Medicine-Their Relationship to Family Medicine Specialty Choice
}

Arch G. Mainous III, PhD; Maribeth Porter, MD, MS; Denny Fe Agana, MPH; Alexander W. Chessman, MD

BACKGROUND AND OBJECTIVES: The United States suffers from a low proportion of medical students pursuing family medicine (FM). Our objective was to examine institutional characteristics consistent with a focus on National Institutes of Health (NIH) research, institutional support for FM education, and the proportion of medical students choosing FM.

METHODS: The 2015 CERA Survey of Family Medicine Clerkship Directors was merged with institutional NIH funding data from 2014 and medical student specialty choice in 2015 . Institutional educational support was operationalized as (1) clerkship director's perception of medical school environment toward FM, and (2) amount of negative comments about FM made by faculty in other departments. The outcome was the percentage of students selecting FM. Bivariate statistics were computed.

RESULTS: As NIH funding increases, the proportion of students entering FM decreases $(r=-.22)$. Institutions with higher NIH funding had lower clerkship director perceptions of medical school support toward FM ( $r=-.38)$. Among private institutions, the negative correlation between NIH funding and the proportion of students entering FM strengthens to $r=-.48, P=.001$. As perceptions of support for FM increase, the proportion of students entering FM increase $(r=.47)$. Among private schools, perceptions of support toward family medicine was strongly positively correlated with the proportion of students entering FM $(r=.72, P=.001)$.

CONCLUSIONS: Higher institutional NIH funding is associated with less support for FM and lower proportions of students choosing FM. These issues appear to be even more influential in private medical schools. Understanding how to integrate the goals of NIH-level research and increasing primary care workforce so that both can be achieved is the next challenge.

(Fam Med. 2018;50(5):369-71.)

doi: 10.22454/FamMed.2018.913629

A debate is currently raging as to whether the United States faces a physician shortage..$^{1,2}$ Some authors suggest that the US needs to produce more physicians, while other projections indicate that the number is sufficient for future needs. Although it may be unclear whether the US faces a physician shortage, it is clear that the US has a relatively low proportion of medical students choosing a career in family medicine. ${ }^{3}$ Having an insufficient number of primary care physicians has been associated with mortality in a health system with universal health care access. ${ }^{4,5}$

Factors related to medical student specialty choice have been evaluated in a wide variety of studies. ${ }^{6}$ One area that has not been closely studied is the influence of the institutional culture on corresponding medical student specialty choice. ${ }^{7}$ Medical school deans must balance the tension between the goal of having a large portfolio of peer-reviewed research funding from the National Institutes of Health (NIH), and the goal of producing primary care practitioners. It is unclear whether these two goals lead to two distinct sets of institutions with different priorities, and how that may affect the corresponding specialty choice of their medical students.

Our objective was to examine the relationship between institutional characteristics consistent with a focus on NIH research and institutional support for family medicine education with the proportion of medical students choosing family medicine.

From the Department of Health Services Research, Management and Policy (Dr Mainous III) and Department of Community Health and Family Medicine, University of Florida, Gainesville (Drs Mainous III, Porter, and Agana); and the Department of Family Medicine, Medical University of South Carolina, Charleston (Dr Chessman). 


\section{Methods}

This study is an analysis of data collected in the national family medicine clerkship directors 2015 survey conducted by the Council of Academic Family Medicine Educational Research Alliance (CERA). ${ }^{8}$ Family medicine clerkship directors at US allopathic medical schools were identified for participation. Because there is no centralized list of clerkship directors, names and contact information of clerkship directors were solicited through communication within the STFM Group on Medical Student Education. There were 125 active unique individuals at US allopathic medical schools with confirmed valid email addresses. The study was approved by the American Academy of Family Physicians Institutional Review Board.

The CERA survey data were merged with data on the specialty choice for each institution's 2015 graduating medical school class. The CERA survey data and specialty choice data were merged with a third source of data, the 2014 amount of NIH funding to each institution in dollars. The deidentified data set was provided to the investigators for analysis.

\section{Variables}

Institutional Support for Family Medicine: Institutional support was operationalized as (a) clerkship director's perception of medical school environment toward family medicine, and (b) amount of negative comments about family medicine that the director discovers. The survey contained the question, "In general, how do you perceive the environment of your medical school towards family medicine?" which was scored on a 5-point scale of 1-very much against ("toxic") to 5 -very much promotes ("embraces"). The second question asked how often the director discovers that a faculty member in another department has made a negative comment about family medicine $(0$, less than 1 per year, about 1 per year, 2 to 5 times per year, or 6 or more times per year).

Public or Private Insititution: The survey contained a question inquiring whether the institution was considered public or private.

NIH Funding: The 2014 total institutional dollar amount of NIH funding to each institution was assessed. This NIH information was obtained from publicly available data at the Blue Ridge Institute for Medical Research (http://www.brimr.org/NIH Awards/2014/NIH_Awards_2014. htm; accessed July 12, 2017).

Specialty Choice of Family Medicine: The proportion of the 2015 graduating medical school class of each institution choosing family medicine was assessed using data publicly available on the American Academy of Family Physicians' website (http://www.aafp.org/medicalschool-residency/faculty/medschools/ schools-complete-list.html; accessed July 12, 2017).

\section{Analysis}

Our analysis was limited to institutions that provided data on all of the investigated variables $(\mathrm{n}=96)$. We calculated descriptive statistics using chi-square tests. We computed Pearson correlations for continuous variables and Spearman's correlations for ordinal variables. All analyses were performed using SAS version 9.4 .

\section{Results}

In terms of the correlations, as NIH funding increases the proportion of students entering family medicine decreases $(r=-.22, P=.03)$. Institutions with higher NIH funding had lower clerkship director perceptions of medical school support toward family medicine $(r=-.38, P=.001)$ and more instances of faculty in other departments disparaging family medicine $(r=-.19, P=.06)$.

When the institutions are separated into public or private, significant differences emerge. Among private institutions, the correlation between $\mathrm{NIH}$ funding and the proportion of students entering family medicine strengthens to $r=-.48, P=.001$. When examining public schools, the correlation between NIH funding and the proportion of students entering family medicine is much weaker and not statistically significant at $r=-.06$, $P=.62$.

Perceptions of support for family medicine and instances of other faculty disparaging family medicine were negatively correlated $(r=-.24$, $P=.02$ ). As perceptions of support for family medicine increased the proportion of students entering family medicine increased ( $r=.47, P=.001$ ). However, there was a weak relationship between number of instances of disparaging remarks and the percentage of students choosing family medicine ( $r=-.04, P=.72$ ).

Among private schools, clerkship director perceptions of medical school support toward family medicine was strongly correlated with the proportion of students entering family medicine $(r=.72, P=.001)$. In public institutions there was a similar direction in the correlation between perception of support and proportion of students entering family medicine, but it was substantially weaker $(r=.37, P=.002$ ). Among private institutions, the correlation between negative comments and proportion of the class choosing family medicine was $r=-.16, P=.43$, while in public schools the correlation was smaller at $r=-.02, P=.83$, yet still not statistically significant.

When the FM clerkship director's ${ }^{1}$ perception was dichotomized, an institution that was perceived as promoting family medicine, rather than against family medicine, had a higher proportion $(11.8 \%, \mathrm{SD}=4.2)$ of students entering family medicine than institutions with perceptions against family medicine $(7.6 \%$, $\mathrm{SD}=3.9, P=.001$ ).

\section{Discussion}

The results of this study indicate that there may be cultural factors within medical schools that impact the goals of being a strong research institution and a school that produces primary care physicians. The 
relationship between NIH funding, perception of support of family medicine, and students choosing family medicine is particularly pronounced in private institutions. These results on NIH and funding and family medicine specialty choice in private schools are similar to results previous studies. ${ }^{9}$ The results suggest that reports of negative comments may not be as strong an indicator of family medicine specialty selection as perceived support for family medicine, ie, the overall positive support was more important than what could be just a few negative comments. A culture focused on NIHlevel research, and perhaps a more elite orientation within the medical school magnifies the impact of perceived support for family medicine and a corresponding lower proportion of students choosing family medicine. Changing culture to accommodate both research and production of a primary care workforce may be difficult but should be an aspirational goal.

There are several limitations to this study. First, the perception of institutional culture by the clerkship director may not be an accurate reflection of attitudes toward family medicine throughout the institution. However, as the primary face of family medicine to medical students, these clerkship directors are likely to hear about negative comments made on other rotations. The measure of reports of negative comments may not be as strong an indicator as perceived support for family medicine as was indicated by the results. Second, while we were concerned with the production of primary care physicians, we only evaluated the specialty choice of family medicine. Other primary care specialties may have different perceptions of support.

In conclusion, medical schools have a responsibility to society to produce primary care physicians. Future research on primary care and population health may help to strengthen this case. Medical schools also seek NIH funding which not only improves their institutional reputation, but also advances medical science through research. The two goals do not need to be mutually exclusive, and medical school administrations should determine how to do both. Encouraging a culture of support for family medicine and primary care and primary care research within the institution may be a strategy to accomplish this.

CORRESPONDING AUTHOR: Address correspondence to Dr Mainous III, Department of Health Services Research, Management, and Policy, University of Florida, Health Sciences Center, PO Box 100195, Gainesville, FL 32610. 352-273-6073. Fax: 352-273-6075. arch.mainous@ufl.edu.

\section{References}

1. Kirch DG, Petelle K. Addressing the physician shortage: the peril of ignoring demography. JAMA. 2017;317(19):1947-1948

2. Gudbranson E, Glickman A, Emanuel EJ. Reassessing the data on whether a physician shortage exists. JAMA. 2017;317(19):19451946.

3. Kozakowski SM, Travis A, Bentley A, Fetter G Jr. Entry of US medical school graduates into family medicine residencies: 2015-2016. Fam Med. 2016;48(9):688-695.

4. Baker R, Honeyford K, Levene LS, et al. Population characteristics, mechanisms of primary care and premature mortality in England: a cross-sectional study. BMJ Open. 2016;6(2):e009981.

5. Jerant A, Fenton JJ, Franks P. Primary care attributes and mortality: a national person-level study. Ann Fam Med. 2012;10(1):34-41.

6. Pfarrwaller E, Audétat MC, Sommer J, et al. An expanded conceptual framework of medical students' primary care career choice. Acad Med. 2017;92(11):1536-1542; Epub ahead of print.

7. Tandeter H, Granek-Catarivas M. Choosing primary care? Influences of medical school curricula on career pathways. Isr Med Assoc J. 2001;3(12):969-972.

8. Mainous AG III, Seehusen D, Shokar N. CAFM Educational Research Alliance (CERA) 2011 Residency Director survey: background, methods, and respondent characteristics. Fam Med. 2012;44(10):691-693.

9. Brode EC, Petterson SM, Bazemore AW. Is NIH research funding to medical schools associated with more family physicians? Am Fam Physician. 2013;87(3):214 